\title{
Detection of the Novel optrA Gene Among Linezolid-Resistant Enterococci in Barcelona, Spain
}

\author{
Jordi Càmara, Mariana Camoez,,2 Fe Tubau, ${ }^{1,3}$ Miquel Pujoll, ${ }^{2,4}$ Josefina Ayats,,3 \\ Carmen Ardanuy, ${ }^{1,3}$ and M. Ángeles Domínguez ${ }^{1,2}$
}

\begin{abstract}
The purpose of this study was to describe the presence of the novel optrA gene among clinical isolates of enterococci in a Spanish teaching hospital (May 2016-April 2017). optrA and cfr genes were screened by PCR in all isolates showing linezolid minimal inhibitory concentration (MIC) $\geq 4 \mathrm{mg} / \mathrm{L}$. The genetic relatedness of the isolates, the presence of resistance and virulence genes, and the genetic environment of optrA were assessed by whole-genome sequencing (WGS). Six of 1,640 enterococci had linezolid MIC $\geq 4 \mathrm{mg} / \mathrm{L}$. Among them, the optrA gene was detected in five Enterococcus faecalis isolated from unrelated patients. Although none of them had received linezolid or chloramphenicol, all had antecedents of recent quinolone consumption. WGS analysis revealed the existence of two different genotypes: ST585 and ST474. $c f r$ was not detected in any of the isolates. No mutations were detected among the 23S ribosomal RNA and the ribosomal proteins L3, L4, and L22. Both genotypes also carried genes related to aminoglycoside, lincosamide, macrolide, phenicol, and tetracycline resistance. Detection of optrA in a setting with low linezolid consumption and among patients without antecedents of oxazolidinone therapy is of concern.
\end{abstract}

Keywords: linezolid, optrA, cfr, enterococci, chloramphenicol, Enterococcus faecalis

\section{Introduction}

$\mathbf{L}$ INEZOLID RESISTANCE IS emerging as a problem in the treatment of multidrug-resistant Gram-positive organisms, particularly methicillin-resistant Staphylococcus aureus and vancomycin-resistant Enterococcus faecium. ${ }^{1,2}$ Among enterococci, linezolid resistance is mainly mediated by chromosomal mutations in domain $\mathrm{V}$ of the $23 \mathrm{~S}$ ribosomal RNA (rRNA). ${ }^{3}$ However, a transferable oxazolidinone resistance gene $(c f r)$, which encodes a methyltransferase that modifies the 23S rRNA, was described in 2001. ${ }^{4}$ This gene confers resistance to linezolid, phenicols, lincosamides, pleuromutilins, and streptogramin A. Recently, a novel mechanism (optrA) was described in Enterococcus spp., ${ }^{5-12}$ Staphylococcus sciuri, ${ }^{13,14}$ and Streptococcus suis, ${ }^{15}$ indicating its ability to spread to different genus. optrA encodes an ABC-F protein that protects the bacterial ribosome from the antibiotic inhibition, has the ability to spread through plasmids, and confers resistance to linezolid and phenicols. 5,16

In our setting, Hospital Universitari de Bellvitge (HUB), linezolid resistance is rare $(<1 \%$ in both enterococci and staphylococci) and the $c f r$ gene has been sporadically detected in $S$. aureus. ${ }^{17}$ The purpose of this study was to screen for the presence of transferable linezolid resistance among linezolid-resistant clinical isolates of enterococci in a tertiary care hospital in Barcelona, Spain.

\section{Materials and Methods}

Study setting, bacterial isolates, and antimicrobial susceptibility testing

This study was performed at HUB, a 700-bed teaching hospital located in the urban area of Barcelona, Spain. From May 2016 to April 2017, all enterococci isolates obtained from clinical samples were routinely identified by MatrixAssisted Laser Desorption/Ionization-Time of Flight Mass Spectrometry (MALDI-TOF MS) (Bruker Daltonik GmbH, Bremen, Germany) and tested for antimicrobial susceptibility by microdilution (Microscan ${ }^{\circledR}$; Beckman Coulter, Brea, CA) following the Clinical and Laboratory Standards Institute (CLSI) recommendations and criteria. ${ }^{18}$ The linezolid minimal inhibitory concentration (MIC) breakpoints were as follows:

\footnotetext{
${ }^{1}$ Microbiology Department, Hospital Universitari de Bellvitge-Universitat de Barcelona-IDIBELL, L'Hospitalet de Llobregat, Barcelona, Spain.

${ }^{2}$ Spanish Network for Research in Infectious Diseases (REIPI), Instituto de Salud Carlos III, Madrid, Spain.

${ }^{3}$ CIBER de Enfermedades Respiratorias (CIBERES), Instituto de Salud Carlos III, Madrid, Spain.

${ }^{4}$ Infectious Diseases Department, Hospital Universitari de Bellvitge-Universitat de Barcelona-IDIBELL, L'Hospitalet de Llobregat, Barcelona, Spain.
} 
susceptible $\leq 2 \mathrm{mg} / \mathrm{L}$, intermediate $4 \mathrm{mg} / \mathrm{L}$, and resistant $\geq 8 \mathrm{mg} /$ L. Isolates showing linezolid MIC $\geq 4 \mathrm{mg} / \mathrm{L}$ were further studied by microdilution, using Sensititre Rapmyco ${ }^{\circledR}$ plates (Thermo Fisher Scientific, Waltham, MA) with linezolid concentrations ranging from 1 to $32 \mathrm{mg} / \mathrm{L}$.

\section{Molecular characterization}

Linezolid-resistant isolates were screened for both $c f r$ and optrA genes by PCR, as described elsewhere. ${ }^{7}$ Wholegenome sequencing (WGS) was performed in those optrApositive isolates. Libraries were prepared (NexteraXT ${ }^{\circledR}$ ) and multiplexed in an MiSeq run (150 bp paired-reads). Reads were processed using Geneious 9.1.7 (Biomatters, Auckland, New Zealand): duplicate reads were removed, ends were trimmed, and reads were de novo assembled using the Geneious assembler with default settings (medium sensitivity). The genetic environment of optrA was in silico studied comparing those contigs containing the optrA gene with those previously published. The existence of resistance and virulence genes and the genetic relatedness of the isolates were studied through the online tools Resfinder 2.1, Virulence finder 1.5, and CSI phylogeny (www .genomicepidemiology.org/, last accessed June 15). This last analysis included, for comparative purposes, all previously sequenced optrA-positive Enterococcus faecalis strains (Supplementary Table S1; Supplementary Data available online at www.libertpub.com/mdr). Data from WGS were also used to search for chromosomal mutations related to oxazolidinone (23S rRNA and ribosomal proteins L3, L4, and L22) and quinolone (GyrA and ParC proteins) resistance. E. faecalis ATCC 29212 (accession number CP008816) was used as reference strain. Raw data were deposited to the European Nucleotide Archive (www.ebi.ac.uk/ena) with accession numbers ERS1788479-1788483.

\section{Clinical data}

Clinical data of patients were retrospectively reviewed from electronic records. Data included age, sex, admission ward, previous antimicrobial therapy, treatment, and outcome. This study was approved by the Clinical Research Ethics Committee of HUB (PR354/17). As no intervention was involved and patients' identification was anonymized, written informed consent was considered not necessary.

\section{Results}

During the study period, 1,640 enterococci isolates were collected from clinical samples of 1,249 patients. Table 1 gives the species and the linezolid MICs of the studied population. Overall, 1,634 isolates $(99.6 \%)$ had $\mathrm{MIC} \leq 2 \mathrm{mg} / \mathrm{L}$ and only 6 E. faecalis $(0.4 \%)$ were linezolid nonsusceptible (MIC $\geq 4 \mathrm{mg} / \mathrm{L}$ ). In our series, E. faecalis isolates had significantly higher linezolid MICs than $E$. faecium $(p<0.01)$. After PCR screening, the optrA gene was detected in five of these nonsusceptible isolates, all showing chloramphenicol MIC of $>256 \mathrm{mg} / \mathrm{L}$ (Table 2). We did not detect the $c f r$ gene in any of the isolates. All five isolates were recovered from urine samples of five male patients admitted to three different wards. Two patients had clinical symptoms of urinary tract infection and were successfully treated with aminopenicillins (ampicillin and amoxicillin). The remaining three patients
Table 1. ENTERococcus SPeCIES AND Linezolid MICs of THE STUdy Population

\begin{tabular}{lrrrr}
\hline & \multicolumn{4}{c}{$\begin{array}{c}\text { Linezolid MIC (mg/L), } \\
\text { number of isolates (\%) }\end{array}$} \\
$\begin{array}{lrrrr}\text { Enterococcus } \\
\text { species }\end{array}$ & \multicolumn{1}{c}{2} & 2 & 4 & $>4$ \\
\hline E. avium & $12(85.7)$ & $2(14.3)$ & & \\
$\begin{array}{l}\text { E. casseliflavus } \\
\text { Enterococcus }\end{array}$ & $2(66.7)$ & $1(33.3)$ & & \\
$\quad$ faecalis & $703(59.0)$ & $483(40.5)$ & $2(0.2)$ & $4(0.3)$ \\
Enterococcus & $289(70.0)$ & $124(30.0)$ & & \\
$\quad$ Faecium & & & & \\
E. gallinarum & $4(66.7)$ & $2(33.3)$ & & \\
E. hirae & $1(25.0)$ & $3(75.0)$ & & \\
E. raffinosus & $4(100)$ & & & \\
Other & $2(50.0)$ & $2(50.0)$ & & \\
All & $1,017(62.0)$ & $617(37.6)$ & $2(0.1)$ & $4(0.2)$ \\
\hline
\end{tabular}

Only species accounting for more than two isolates are given.

CLSI linezolid MIC breakpoints were as follows: susceptible $\leq 2 \mathrm{mg} / \mathrm{L}$, intermediate $4 \mathrm{mg} / \mathrm{L}$, and resistant $\geq 8 \mathrm{mg} / \mathrm{L} .^{18}$

MIC, minimal inhibitory concentration; CLSI, Clinical and Laboratory Standards Institute.

were asymptomatic and their isolates were obtained from low urinary bacterial counts. Consequently, these isolates were considered colonizing and the patients did not receive antimicrobial therapy. As we did not find any epidemiological relationships between these five patients, these acquisitions were considered unrelated. Although none of them had received oxazolidinones or phenicols, all had antecedents of recent ( $<6$ months) antimicrobial therapy.

As deduced from WGS, isolates belonged to two sequence types (ST474 and ST585) that were also discriminated by the single-nucleotide polymorphism (SNP) analysis: SNP differences were 502 among ST474 isolates, ranged from 140 to 300 among ST585 isolates, and were $\sim 15,000$ between both groups (Supplementary Table S2). Overall, the WGS analysis of E. faecalis carrying optrA displayed high genetic diversity, including, as defined by multilocus sequence typing (MLST), 12 different genotypes (Fig. 1 and Supplementary Table S1). With regard to the isolates presented in this study, although ST585 strains carrying optrA have been described from human samples in China, ${ }^{5,6}$ we could not find any report of ST474. This sequence type that was added to the MLST webpage (www.mlst.net) from the Chinese Guangdong province (liver sample from a pig) shares four of seven MLST alleles with ST59. Interestingly, although this ST59 was first reported from a Spanish pig in $2001,{ }^{19}$ it has been recently detected among optrA-positive isolates in China (human and animal sources) $^{5,6}$ and Colombia (poultry meat). ${ }^{8}$ Data from WGS confirmed this relationship showing that ST474 isolates were related to the ST59 Colombian isolates (12E and 34E) and to an additional isolate collected in Malaysia in 2012 (Enfs85).

Investigation of acquired resistance genes revealed that all isolates carried genes encoding resistance to tetracyclines, macrolides, lincosamides, streptogramin B, and phenicols (Table 2). Four isolates shared the OptrA wild type, originally described in E. faecalis E394, whereas one ST474 isolate had an amino acid substitution (Tyr176Asp). ${ }^{6}$ All strains harbored cat (cat A-8) and fexA genes, both related 


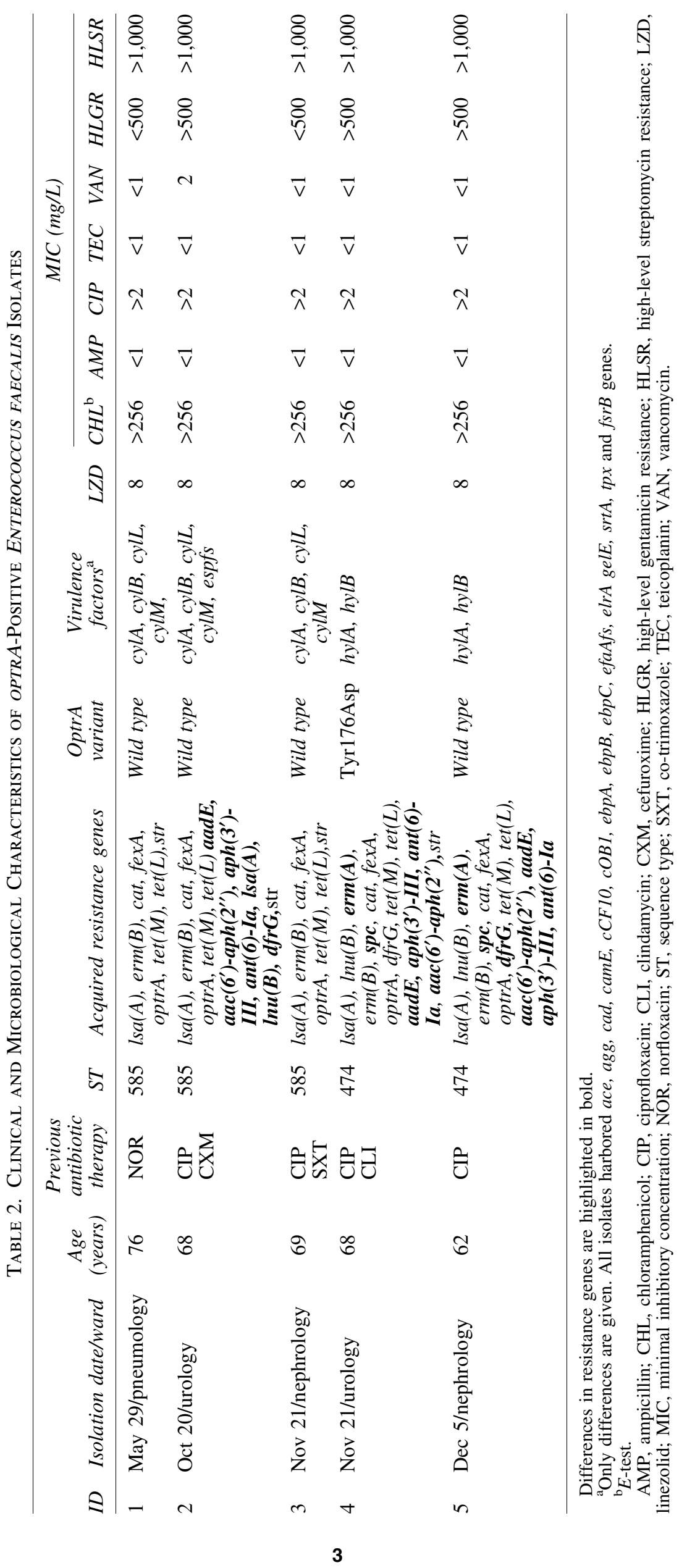


FIG. 1. SNP phylogenetic tree of Enterococcus faecalis carrying optrA. The scale bar represents the genetic divergence between isolates (number of SNPs/total number of polymorphic sites). The figure includes isolates reported in this study (isolates 1-5), previously published isolates, and WGS sequences of optrA-positive E. faecalis obtained after performing a blast search in the NCBI public database (Table S1). SNP, singlenucleotide polymorphism; WGS, whole-genome sequencing.

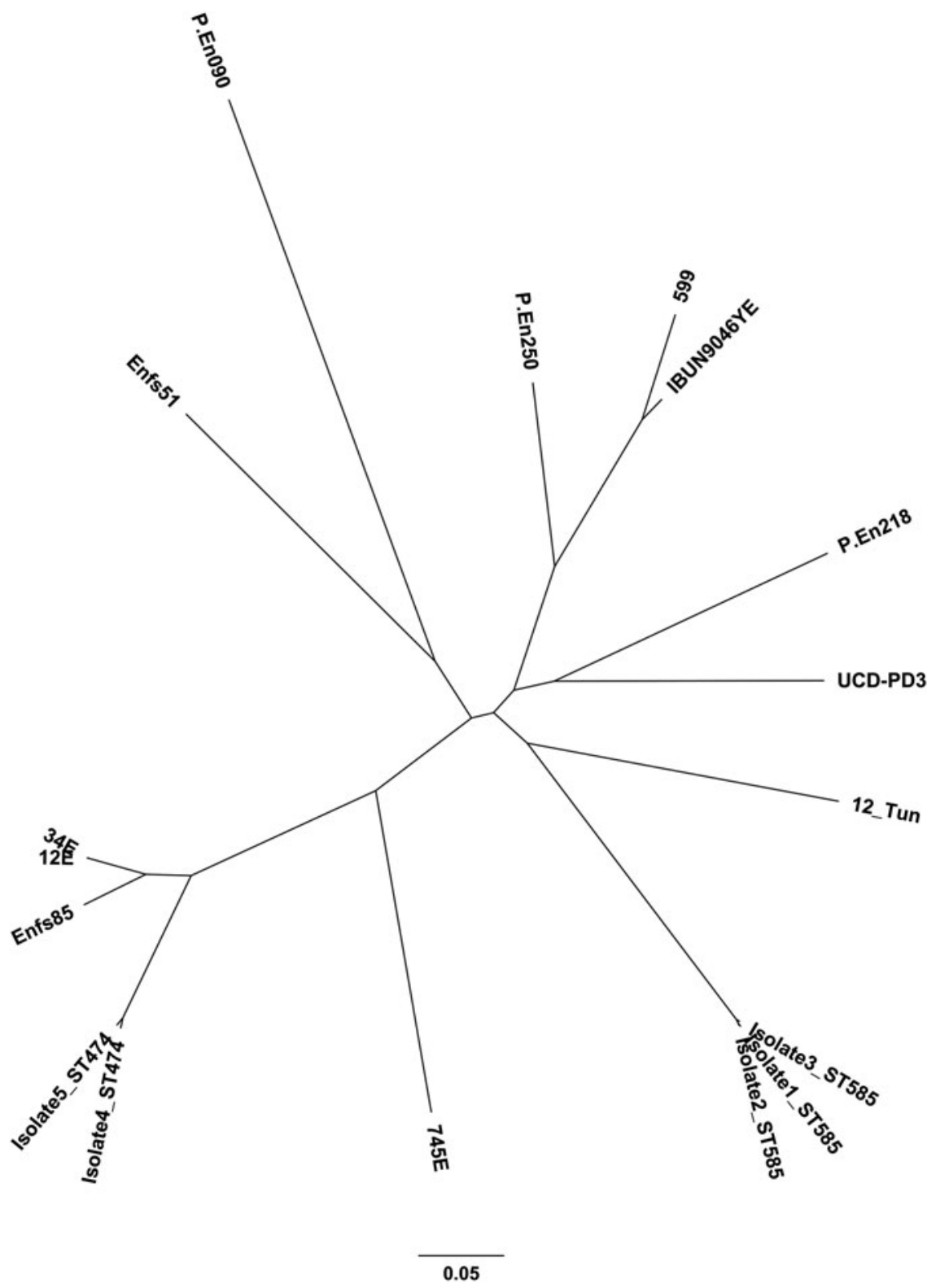

to phenicol resistance. No mutations were detected among the 23S rRNA and ribosomal proteins L3, L4, and L22. One ST585 isolate and all ST474 isolates carried the $a a c\left(6^{\prime}\right)$ $\operatorname{aph}\left(2^{\prime \prime}\right)$ gene, conferring high-level resistance to gentamicin. All isolates showed amino acid substitutions related to quinolone resistance ${ }^{20}$ in ParC (Ser80Ile) and GyrA (Ser83Phe and Glu87Gly in ST585 and ST474 isolates, respectively). Both genotypes shared a high number of virulence factors including genes related to adhesion (ace, agg, efaAfs, and $e l r A)$, biofilm production ( $e b p A, e b p B$, and $e b p C$ ), proteases ( $\mathrm{gelE}$ and $s r t A$ ), protection against oxidative stress (tpx), and genes involved in quorum sensing ( $\mathrm{cad}$, camE, cCF10, cOB1, and $f s r B$ ). Main differences were the existence of the cytolysin operon ( $c y l A, c y l B, c y l L$, and $c y l M$ genes) only in ST585 and the presence of hyaluronidase genes hylA and hylB only in ST474. As both STs were responsible for episodes of not only infection but also colonization, we could not hypothesize about the importance of these factors in the pathogenesis of isolates.
Finally, the genetic environment of the optrA gene was in silico compared with previously published data and, in agreement with that, all ST585 isolates harbored a genetic context identical to that reported from plasmid pXY17 (described in ST585 isolates from China). Isolate 4 (ST474) showed a structure related to plasmid p10-2-2, which is in agreement with the fact that this plasmid was found in an ST59 isolate, genetically related to ST474. ${ }^{10}$ Isolate 5 showed truncated contigs and the region could only be partially revealed (Fig. 2).

\section{Discussion}

The frequent detection of optrA among Chinese isolates of animal origin suggests their selection by the use of antibiotics in animals (florfenicol), or indirectly by other antibiotics for human uses whose resistance mechanisms are harbored by the same plasmids. A previous study found a high prevalence of linezolid resistance genes in soils next to 


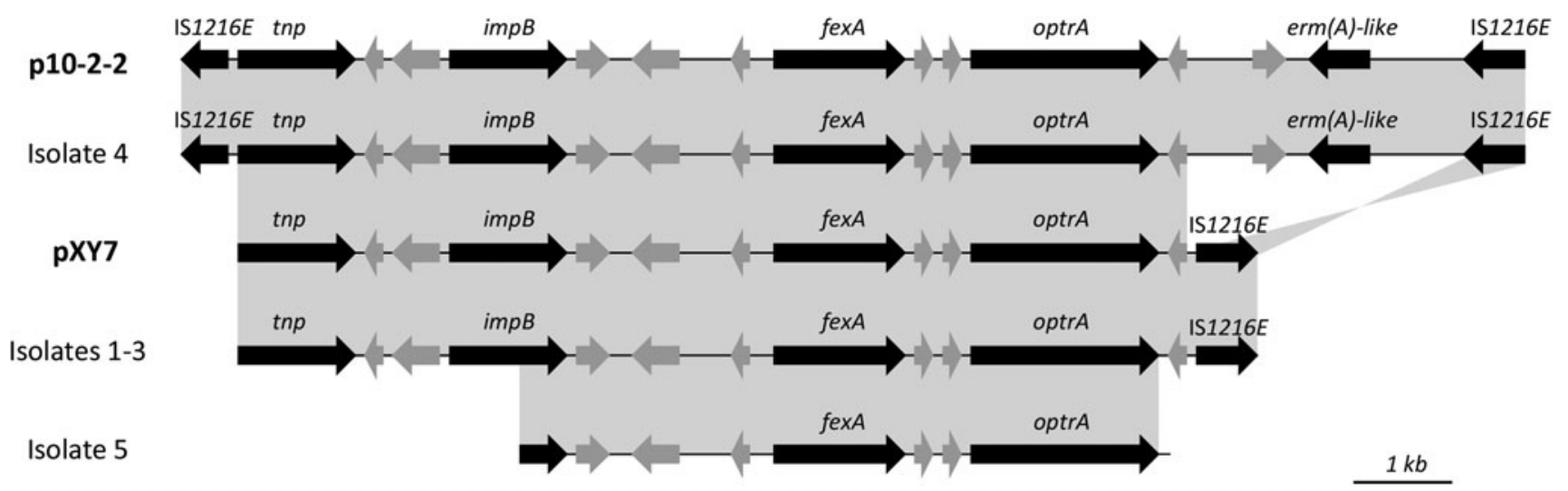

FIG. 2. Graphic representation of the genetic environment of optrA containing regions. Contigs containing optrA were compared with previously published regions and then reads were mapped against these regions. Isolates 1, 2, and 3 (ST585) shared the same genetic environment described in plasmid pXY17 and isolate 4 (ST474) showed a structure related to plasmid p10-2-2. The structure of the region containing optrA of isolate 5 could only be partially revealed.

pig farms in China, including both $c f r$ and optrA genes, establishing a clear link between resistance and livestock farming. ${ }^{21}$ In addition, the recent report of optrA from a human-related lineage of E. faecalis (ST86) in urban wastewater (Tunisia) suggests the existence of effective dissemination in the human setting. ${ }^{22}$ Data from European countries and the United States are still scarce. In Europe, a few optrApositive enterococci isolates have been reported. ${ }^{7,11,12,23} \mathrm{~A}$ recent multicenter study carried out on isolates from 42 countries, excluding the United States, detected the optrA gene in 9 isolates ( 8 E. faecalis and $1 \mathrm{~S}$. gallolyticus) from a large sample of 854 enterococci and 469 viridans-group streptococci screened. ${ }^{24}$ In a linezolid surveillance program in the United States, the optrA gene was found in 2 E. faecalis of 973 enterococci screened. ${ }^{25}$

This is the first detection of optrA in Spain, as far as is known. As linezolid consumption is low in our setting, and none of the patients harboring optrA-positive isolates received linezolid or chloramphenicol therapy, our findings are of concern. As reported, the coexistence of other resistance markers in the same mobile genetic elements could justify the role of antimicrobials other than oxazolidinones or phenicols in spreading these strains. ${ }^{5}$ Although we were not able to find optrA and other resistance genes colocated in the same genetic environment (with the exception of $f e x A$ ), their association cannot be excluded from our data. Therefore, other antimicrobials for human use (i.e., macrolides, quinolones, tetracyclines, or streptomycin) could contribute to the selection of these isolates in the clinical setting. In this respect, it is remarkable that all isolates were resistant to ciprofloxacin and this was because of a nontransferable mechanism. Data on antibiotic consumption from our hospital indicate that, after penicillins, quinolones are the second group of most prescribed antimicrobials (10.74 prescribed daily doses per 100 patient-days in 2015), substantially exceeding the consumption of linezolid ( 0.48 daily doses per patient-day in 2015). The fact that all isolates were recovered from urine samples and all patients had antecedents of quinolones consumption, which are frequently used in urine tract infections, could indicate that, in our clinical setting, these antibiotics may have played an important role in the selection of these isolates. In fact, quinolones have already been in- volved in mechanisms of antimicrobial resistance co-selection among Gram-negative bacteria. ${ }^{26}$ Although optrA has not been clearly linked to any case of clinical failure after linezolid treatment, its presence in the hospital setting is worrisome. As no evidence of chromosomal mutations related to linezolid resistance were detected (that are usually associated to linezolid utilization), ${ }^{27}$ it seems that the emergence of optrA isolates in our setting is independent of oxazolidinone consumption. In addition, the detection of different genotypes isolated from apparently unrelated patients could be an indirect sign of its dissemination in our region and beyond the hospital setting, which needs to be clarified in further studies.

Our study adds epidemiological information on transferable linezolid resistance. Data obtained from WGS are particularly useful for assessing genetic relatedness among bacteria, allowing data sharing worldwide. The study of previously published WGS sequences of optrA-positive E. faecalis reveals the existence of multiple lineages and worldwide distribution. Specifically, WGS allowed us to put in context our clinical isolates and to establish genetic relationship between the two of them (isolates 4 and 5), an isolate from animal origin (Malaysia, 2012) and two isolates from poultry meat (Colombia, 2011). ${ }^{8}$ WGS is not able to solve the origin of this association. However, the fact that these isolates were obtained from both human and animal origin in several different countries indicates the existence of a wide distribution of optrA. As isolates belonging to ST59 have been described before the optrA description in animals ${ }^{19,28}$ and also in the clinical setting, ${ }^{29}$ the ability of this lineage to cause invasive human disease is evident. Actually, this goes in line with the high number of virulence factors detected, most of them also shared by ST585 isolates.

Our study has limitations. As we only included isolates showing linezolid MIC $\geq 4 \mathrm{mg} / \mathrm{L}$, and optrA has been detected in isolates with lower MICs, ${ }^{5,7,9,13}$ our findings could underestimate its presence. This could be especially important in environments with higher linezolid consumption and higher resistance rates that could masquerade the presence of this mechanism. In addition, although WGS is a powerful epidemiological tool, complete plasmid sequences are difficult to assemble through short reads giving only partial results that makes the analysis of these genetic elements difficult. ${ }^{30}$ 
In conclusion, we report the presence of optrA among clinical linezolid-resistant isolates in an urban area of Barcelona that is not related to linezolid consumption. Active surveillance and continued efforts to control the use of antimicrobials in veterinary and clinical practice are needed worldwide. Otherwise, the arsenal of available antimicrobial drugs against infections caused by multidrug resistance could be significantly reduced.

\section{Acknowledgments}

The authors thank the staff at the Microbiology Department of Hospital Universitari de Bellvitge for their daily contributions to this study.

The study was supported by Plan Nacional de I+D+i and Instituto de Salud Carlos III, Subdirección General de Redes y Centros de Investigación Cooperativa, Ministerio de Economía y Competitividad, Spanish Network for the Research in Infectious Diseases (REIPI RD12/0015)co-financed by European Development Regional Fund "A way to achieve Europe" ERDF, and partially supported by Ciber de Enfermedades Respiratorias (CB06/06/0037 Ministry of Health, Instituto de Salud Carlos III, Madrid, Spain).

\section{Disclosure Statement}

No competing financial interests exist.

\section{References}

1. Tsiodras, S., H.S. Gold, G. Sakoulas, G.M. Eliopoulos, C. Wennersten, L. Venkataraman, R.C. Moellering, and M.J. Ferraro. 2001. Linezolid resistance in a clinical isolate of Staphylococcus aureus. Lancet. 358:207-208.

2. Gonzales, R.D., P.C. Schreckenberger, M.B. Graham, S. Kelkar, K. DenBesten, and J.P. Quinn. 2001. Infections due to vancomycin-resistant Enterococcus faecium resistant to linezolid. Lancet. 357:1179.

3. Mendes, R.E., L.M. Deshpande, and R.N. Jones. 2014. Linezolid update: stable in vitro activity following more than a decade of clinical use and summary of associated resistance mechanisms. Drug Resist. Updat. 17:1-12.

4. Schwarz, S., C. Werckenthin, and C. Kehrenberg. 2000. Identification of a plasmid-borne chloramphenicol-florfenicol resistance gene in Staphylococcus sciuri. Antimicrob. Agents Chemother. 44:2530-2533.

5. Wang, Y., Y. Lv, J. Cai, S. Schwarz, L. Cui, Z. Hu, R. Zhang, J. Li, Q. Zhao, T. He, D. Wang, Z. Wang, Y. Shen, Y. Li, A.T. Fessler, C. Wu, H. Yu, X. Deng, X. Xia, and J. Shen. 2015. A novel gene, optrA that confers transferable resistance to oxazolidinones and phenicols and its presence in Enterococcus faecalis and Enterococcus faecium of human and animal origin. J. Antimicrob. Chemother. 70: 2182-2190.

6. Cai, J., Y. Wang, S. Schwarz, H. Lv, Y. Li, K. Liao, S. Yu, K. Zhao, D. Gu, X. Wang, R. Zhang, and J. Shen. 2015. Enterococcal isolates carrying the novel oxazolidinone resistance gene optrA from hospitals in Zhejiang, Guangdong, and Henan, China, 2010-2014. Clin. Microbiol. Infect. 21:1095. e1091-e1094.

7. Brenciani, A., G. Morroni, C. Vincenzi, E. Manso, M. Mingoia, E. Giovanetti, and P.E. Varaldo. 2016. Detection in Italy of two clinical Enterococcus faecium isolates carrying both the oxazolidinone and phenicol resistance gene
optrA and a silent multiresistance gene cfr. J. Antimicrob. Chemother. 71:1118-1119.

8. Cavaco, L.M., J.F. Bernal, E. Zankari, M. Leon, R.S. Hendriksen, E. Perez-Gutierrez, F.M. Aarestrup, and P. Donado-Godoy. 2016. Detection of linezolid resistance due to the optrA gene in Enterococcus faecalis from poultry meat from the American continent (Colombia). J. Antimicrob. Chemother. 72:678-683.

9. Cui, L., Y. Wang, Y. Lv, S. Wang, Y. Song, Y. Li, J. Liu, F. Xue, W. Yang, and J. Zhang. 2016. Nationwide surveillance of novel oxazolidinone resistance gene optrA in Enterococcus isolates in China from 2004 to 2014. Antimicrob. Agents Chemother. 60:7490-7493.

10. He, T., Y. Shen, S. Schwarz, J. Cai, Y. Lv, J. Li, A.T. Fessler, R. Zhang, C. Wu, J. Shen, and Y. Wang. 2016. Genetic environment of the transferable oxazolidinone/ phenicol resistance gene optrA in Enterococcus faecalis isolates of human and animal origin. J. Antimicrob. Chemother. 71:1466-1473.

11. Gawryszewska, I., D. Zabicka, W. Hryniewicz, and E. Sadowy. 2017. Linezolid-resistant enterococci in Polish hospitals: species, clonality and determinants of linezolid resistance. Eur. J. Clin. Microbiol. Infect. Dis. 36:12791286.

12. Mendes, R.E., P.A. Hogan, R.N. Jones, H.S. Sader, and R.K. Flamm. 2016. Surveillance for linezolid resistance via the $\operatorname{Zyvox}(\mathrm{R})$ Annual Appraisal of Potency and Spectrum (ZAAPS) programme (2014): evolving resistance mechanisms with stable susceptibility rates. J. Antimicrob. Chemother. 71:1860-1865.

13. Fan, R., D. Li, Y. Wang, T. He, A.T. Fessler, S. Schwarz, and C. Wu. 2016. Presence of the optrA gene in methicillinresistant Staphylococcus sciuri of porcine origin. Antimicrob. Agents Chemother. 60:7200-7205.

14. Li, D., Y. Wang, S. Schwarz, J. Cai, R. Fan, J. Li, A.T. Fessler, R. Zhang, C. Wu, and J. Shen. 2016. Co-location of the oxazolidinone resistance genes optrA and cfr on a multiresistance plasmid from Staphylococcus sciuri. J. Antimicrob. Chemother. 71:1474-1478.

15. Huang, J., L. Chen, Z. Wu, and L. Wang. 2017. Retrospective analysis of genome sequences revealed the wide dissemination of optrA in Gram-positive bacteria. J. Antimicrob. Chemother. 72:614-616.

16. Sharkey, L.K., T.A. Edwards, and A.J. O'Neill. 2016. ABC-F proteins mediate antibiotic resistance through ribosomal protection. mBio. 7:e01975.

17. Sierra, J.M., M. Camoez, F. Tubau, O. Gasch, M. Pujol, R. Martin, and M.A. Domínguez. 2013. Low prevalence of Cfrmediated linezolid resistance among methicillin-resistant Staphylococcus aureus in a Spanish hospital: case report on linezolid resistance acquired during linezolid therapy. PLoS One. 8:e59215.

18. Clinical and Laboratory Standards Institute. 2017. Performance standards for antimicrobial susceptibility testing; 27th informational supplement. CLSI document M100-S27. CLSI, Wayne, PA.

19. Ruiz-Garbajosa, P., M.J. Bonten, D.A. Robinson, J. Top, S.R. Nallapareddy, C. Torres, T.M. Coque, R. Canton, F. Baquero, B.E. Murray, R. del Campo, and R.J. Willems. 2006. Multilocus sequence typing scheme for Enterococcus faecalis reveals hospital-adapted genetic complexes in a background of high rates of recombination. J. Clin. Microbiol. 44:2220-2228.

20. Yasufuku, T., K. Shigemura, T. Shirakawa, M. Matsumoto, Y. Nakano, K. Tanaka, S. Arakawa, M. Kawabata, 
and M. Fujisawa. 2011. Mechanisms of and risk factors for fluoroquinolone resistance in clinical Enterococcus faecalis isolates from patients with urinary tract infections. J. Clin. Microbiol. 49:3912-3916.

21. Zhao, Q., Y. Wang, S. Wang, Z. Wang, X.D. Du, H. Jiang, X. Xia, Z. Shen, S. Ding, C. Wu, B. Zhou, Y. Wu, and J. Shen. 2016. Prevalence and abundance of florfenicol and linezolid resistance genes in soils adjacent to swine feedlots. Sci. Rep. 6:32192.

22. Freitas, A.R., H. Elghaieb, R. Leon-Sampedro, M.S. Abbassi, C. Novais, T.M. Coque, A. Hassen, and L. Peixe. 2017. Detection of optrA in the African continent (Tunisia) within a mosaic Enterococcus faecalis plasmid from urban wastewaters. J. Antimicrob. Chemother. 72:3245-3251.

23. Vorobieva, V., L. Roer, U.S. Justesen, F. Hansen, N. FrimodtMøller, H. Hasman, and A.M. Hammerum. 2017. Detection of the optrA gene in a clinical ST16 Enterococcus faecalis isolate in Denmark. J. Glob. Antimicrob. Resist. 10:12-13.

24. Mendes, R.E., L. Deshpande, J.M. Streit, H.S. Sader, M. Castanheira, P.A. Hogan, and R.K. Flamm. 2018. ZAAPS programme results for 2016: an activity and spectrum analysis of linezolid using clinical isolates from medical centres in 42 countries. J. Antimicrob. Chemother. 73:1880-1887.

25. Flamm, R.K., R.E. Mendes, P.A. Hogan, J.M. Streit, J.E. Ross, and R.N. Jones. 2016. Linezolid surveillance results for the United States (LEADER surveillance program 2014). Antimicrob. Agents Chemother. 60:2273-2280.

26. Nseir, S., C. Di Pompeo, S. Soubrier, P. Delour, H. Lenci, M. Roussel-Delvallez, T. Onimus, F. Saulnier, D. Mathieu, and A. Durocher. 2005. First-generation fluoroquinolone use and subsequent emergence of multiple drug-resistant bacteria in the intensive care unit. Crit. Care Med. 33:283-289.
27. Mulanovich, V.E., M.D. Huband, S.P. Mccurdy, M.M. Lemmon, M. Lescoe, Y. Jiang, K.V. Rolston, and P.R. LaSala. 2010. Emergence of linezolid-resistant coagulase-negative Staphylococcus in a cancer centre linked to increased linezolid utilization. 2001-2004. J. Antimicrob. Chemother. 65: 2001-2004.

28. Freitas, A.R., C. Novais, P. Ruiz-Garbajosa, T.M. Coque, and L. Peixe. 2009. Clonal expansion within clonal complex 2 and spread of vancomycin-resistant plasmids among different genetic lineages of Enterococcus faecalis from Portugal. J. Antimicrob. Chemother. 63:1104-1111.

29. Kawalec, M., Z. Pietras, E. Daniłowicz, A. Jakubczak, M. Gniadkowski, W. Hryniewicz, and R.J. Willems. 2007. Clonal structure of Enterococcus faecalis isolated from Polish hospitals: characterization of epidemic clones. J. Clin. Microbiol. 45:147-153.

30. Arredondo-Alonso, S., R.J. Willems, W. van Schaik, and A.C. Schurch. 2017. On the (im)possibility of reconstructing plasmids from whole-genome short-read sequencing data. Microb. Genom. 3:e000128.

Address correspondence to: M. Ángeles Domínguez, $M D, P h D$ Microbiology Department Hospital Universitari de Bellvitge-Universitat de Barcelona-IDIBELL Feixa Llarga $s / n$ L'Hospitalet de Llobregat Barcelona 08907

Spain

E-mail: adominguez@bellvitgehospital.cat 